\title{
DETERMINAÇÃO DA ESPESSURA DA CAMADA POLIMÉRICA DE FASES ESTACIONÁRIAS IMOBILIZADAS PARA CROMATOGRAFIA LÍQUIDA DE ALTA EFICIÊNCIA POR TERMOGRAVIMETRIA
}

\author{
Anizio M. Faria*, Cristien A. Marcos, Camila R. M. Vigna e Carol H. Collins \\ Instituto de Química, Universidade Estadual de Campinas, CP 6154, 13084-971 Campinas - SP, Brasil
}

Recebido em 13/2/07; aceito em 19/7/07; publicado na web em 26/2/08

\begin{abstract}
DETERMINATION OF POLYMERIC LAYER THICKNESS ON IMMOBILIZED STATIONARY PHASES FOR HIGHPERFORMANCE LIQUID CHROMATOGRAPHY USING THERMOGRAVIMETRIC ANALYSES. This paper presents a simple and practical thermogravimetric method for determining the layer thickness of immobilized polymer stationary phases used in reversed-phase high-performance liquid chromatography. In this method, the weight loss of different polysiloxanes immobilized onto chromatographic supports, determined over the temperature range $150-650{ }^{\circ} \mathrm{C}$, demonstrated excellent agreement with the sum of carbon and hydrogen content obtained by elemental analysis. The results presented here suggest that the thermogravimetric procedure is an accurate and precise method to determine the polymeric material content on polymer-coated stationary phases.
\end{abstract}

Keywords: stationary phases; thermogravimetric analyses; high-performance liquid chromatography.

\section{INTRODUÇÃO}

A utilização de métodos físico-químicos para a caracterização de fases estacionárias empregadas na cromatografia líquida de alta eficiência (CLAE) é de extrema importância para uma melhor compreensão do mecanismo de retenção dos compostos. Nas fases estacionárias reversas (FR), o mecanismo de retenção cromatográfica é governado essencialmente pelo componente hidrofóbico, isto é, os ligantes orgânicos nas fases quimicamente ligadas (FEQL) ${ }^{1}$ ou polímeros orgânicos nas fases com camadas poliméricas imobilizadas sobre o suporte cromatográfico ${ }^{2,3}$. Sendo assim, as principais características deste componente hidrofóbico, tais como a concentração superficial dos ligantes/espessura da camada polimérica, a eficácia do processo de ligação/imobilização e a seletividade da coluna, precisam ser determinadas.

A análise elementar é o método mais comum para a determinação da porcentagem de carbono de uma fase estacionária ${ }^{4-6}$. Neste método, uma pequena porção da fase é submetida à combustão em presença de oxigênio, resultando em $\mathrm{CO}_{2}$ e $\mathrm{H}_{2} \mathrm{O}$. Estes produtos da combustão são então quantificados e o material orgânico, presente na fase estacionária, é expresso em termos de carbono e hidrogênio. Apesar de ampla utilização, a análise elementar possui uma limitação importante, pois considera-se que todo o carbono e hidrogênio quantificados são originários do conteúdo orgânico da fase. No entanto, em temperaturas elevadas, grupos hidroxilas da sílica são desidratados, liberando água ${ }^{7}$. Além disso, moléculas de água ou de solventes adsorvidas na superfície da fase estacionária irão proporcionar um acréscimo significativo de hidrogênio e carbono nos resultados finais, superestimando a quantidade de material orgânico ligado/imobilizado na fase estacionária.

Uma forma alternativa de se determinar o conteúdo de carbono de uma fase estacionária é através de termogravimetria $(\mathrm{TG})^{8,9}$. As medidas por TG são baseadas na combustão da fase estacionária através do aumento gradual da temperatura ambiente até uma temperatura desejada. Os produtos da combustão vão sendo volatilizados à medida que se aumenta a temperatura, ocorrendo perdas de mas-

*e-mail: anizio@iqm.unicamp.br sa da amostra inicial que, por sua vez, são usadas para calcular a porcentagem de carbono. A principal vantagem da TG em relação à análise elementar é que estas perdas de massa podem ser determinadas sob faixas específicas de temperaturas. Desta forma, conhecendo-se as faixas de temperaturas de ebulição dos solventes (eventualmente presentes na superfície da fase estacionária) e de condensação dos silanóis, falsas contribuições poderão ser eliminadas no cálculo da quantidade de material orgânico originalmente presente no suporte cromatográfico. No entanto, para que a TG possa ser usada para tal finalidade é necessário que a estrutura química do ligante/polímero da fase estacionária seja conhecida ${ }^{9}$.

Neste trabalho foi desenvolvida uma metodologia baseada na termogravimetria para a determinação da porcentagem de carbono em fases estacionárias com polissiloxanos de diferentes comprimentos de cadeias [poli(metiloctilsiloxano) (PMOS), poli(metiltetradecilsiloxano) (PMTDS) e poli(metiloctadecilsiloxano) (PMODS)] imobilizados termicamente sobre suportes de sílica nua e sílica-zirconizada. A partir dos resultados obtidos de porcentagens de carbono dos materiais imobilizados e dos valores de área superficial dos suportes cromatográficos foram determinadas também as espessuras de camada polimérica de cada fase estacionária avaliada.

\section{PARTE EXPERIMENTAL}

\section{Reagentes}

Os solventes empregados, clorofórmio e hexano, ambos de grau cromatográfico, foram adquiridos da Tedia (Rio de Janeiro, Brasil) e foram usados sem prévia purificação. Os suportes cromatográficos foram constituídos de partículas de $5 \mu \mathrm{m}$ de sílica cromatográfica Kromasil (Akzo Nobel, Bohus, Suécia) com tamanho médio de poro de $11,1 \mathrm{~nm}$ e área superficial específica de $309 \mathrm{~m}^{2} \mathrm{~g}^{-1} \mathrm{e}$, também, de partículas esféricas de $5 \mu \mathrm{m}$ de sílica-zirconizada contendo $\sim 21 \%$ de $\mathrm{Zr}$ quimicamente ligado à superfície da sílica Kromasil, diâmetro médio de poro de $8 \mathrm{~nm}$ e área superficial específica de $336 \mathrm{~m}^{2} \mathrm{~g}^{-1}$, as quais foram preparadas de acordo com a referência 10. Os polímeros estudados; poli(metiloctilsiloxano) (PMOS), poli(metiltetradecilsiloxano) (PMTDS) e poli(metiloctadecisiloxano) (PMODS) foram adquiridos da Petrarch/Hüls America (Piscataway, NJ, EUA). 


\section{Preparo das fases estacionárias}

Porções dos suportes cromatográficos (sílica e sílicazirconizada) previamente ativadas em estufa por $12 \mathrm{~h}$ a $140{ }^{\circ} \mathrm{C}$ foram adicionadas a soluções $10 \%(\mathrm{~m} / \mathrm{v})$ dos polímeros (PMOS, PMTDS e PMODS) em hexano, obtendo massas específicas ($\mathrm{m}_{\text {polímero }}$ ) de $0,8 \mathrm{~g}$, i.e. $0,8 \mathrm{~g}$ de polímero para cada grama de suporte. Estas misturas foram agitadas brandamente por $3 \mathrm{~h}$ à temperatura ambiente. Logo após, as soluções foram colocadas em uma capela, sem agitação, à temperatura ambiente por 6 dias para a sorção do polímero ao suporte e evaporação do hexano. Os materiais secos [Si(PMOS), Si(PMTDS), Si(PMODS) e Si-Zr(PMTDS)] foram colocados separadamente em tubos de aço inox $(150 \times 10$ $\mathrm{mm}$ de d.i.), sendo retidos por filtros e conectores. Estes tubos contendo as fases estacionárias foram colocados em um forno tubular e submetidos à imobilização térmica dos polímeros sobre os suportes, sob atmosfera inerte. Cada material foi submetido a um procedimento previamente otimizado para a imobilização dos polímeros ao suporte, conforme é mostrado na Tabela 1 .

Tabela 1. Condições de imobilização térmica dos diferentes polímeros sobre os suportes cromatográficos

\begin{tabular}{lcc}
\hline Fases estacionárias & \multicolumn{2}{c}{ Imobilização térmica } \\
& Temperatura $\left({ }^{\circ} \mathrm{C}\right)$ & Tempo $(\mathrm{h})$ \\
\hline Si(PMOS) & 120 & 8 \\
Si(PMTDS) & 120 & 16 \\
Si(PMODS) & 140 & 8 \\
Si-Zr(PMTDS) & 100 & 12 \\
\hline
\end{tabular}

Após a imobilização térmica, os tubos de aço inox contendo as fases estacionárias foram colocados dentro de um forno, a $50{ }^{\circ} \mathrm{C}$, e conectados a uma bomba cromatográfica Waters 510 (Milford, MA, EUA) para a extração do polímero não-imobilizado. Para tal, foi empregado o hexano como solvente de extração, sendo eluído através do tubo a uma vazão de $0,5 \mathrm{~mL} \mathrm{\operatorname {min } ^ { - 1 }}$ por $4 \mathrm{~h}$.

\section{Caracterização físico-química}

\section{Termogravimetria}

Amostras de aproximadamente $10 \mathrm{mg}$ das diferentes fases estacionárias contidas em cadinhos de platina foram pesadas e então aquecidas à partir da temperatura ambiente $\left(\sim 25^{\circ} \mathrm{C}\right)$ até $1.000^{\circ} \mathrm{C}$ empregando uma razão de aquecimento de $10{ }^{\circ} \mathrm{C} \mathrm{min}^{-1} \mathrm{sob}$ atmosfera dinâmica de ar $\left(50 \mathrm{~mL} \mathrm{~min}^{-1}\right)$. Todas as medidas foram realizadas em um analisador termogravimétrico de alta resolução, TA Instruments modelo TGA-2050 (New Castle, DE, EUA).

\section{Análise elementar}

Aproximadamente $10 \mathrm{mg}$ de cada amostra de fase estacionária foram submetidas a análise elementar para determinação da \% C para comparação com os valores obtidos pela termogravimetria. As medidas foram realizadas em um analisador Perkin-Elmer modelo CHN-2400 (Shelton, CT, EUA).

\section{RESULTADOS E DISCUSSÃO}

Todas as amostras, antes de serem analisadas por termogravimetria, foram submetidas a uma etapa de secagem a $80^{\circ} \mathrm{C}$ por $4 \mathrm{~h}$ para que perdas de massa devido a possíveis resíduos de solventes não interferissem nas medidas. Após esta etapa, as porcentagens de carbono das quatro fases estacionárias foram determinadas a partir das respectivas medidas por termogravimetria. As fases estacionárias analisadas baseiam-se na imobilização térmica de polissiloxanos de diferentes comprimentos de cadeia lateral (Figura 1); PMOS, PMTDS e PMODS, contendo oito, quatorze e dezoito átomos de carbono em cada monômero, respectivamente; sobre dois diferentes suportes cromatográficos, sílica nua e sílica zirconizada. Para a quantificação do conteúdo de carbono de cada uma das fases estacionárias foi necessário a priori determinar as faixas de temperaturas em que ocorre a degradação de cada um dos diferentes polissiloxanos. Para tal, foram obtidas as curvas TG de três amostras de cada polímero puro. A Figura 2 apresenta o perfil de degradação térmica dos polissiloxanos ao longo da faixa de temperatura estudada.

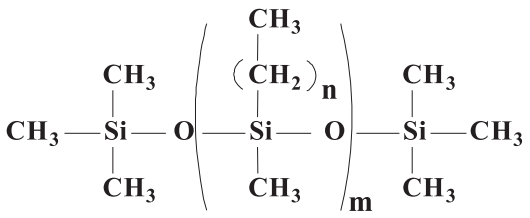

Figura 1. Estrutura química de um poli(metilalquilsiloxano), onde n se refere ao tamanho da cadeia lateral do polímero (para PMOS, $n=7$; PMTDS, $n=$ 13 e PMODS, $n=17$ ) e m é o número de unidades do monômero

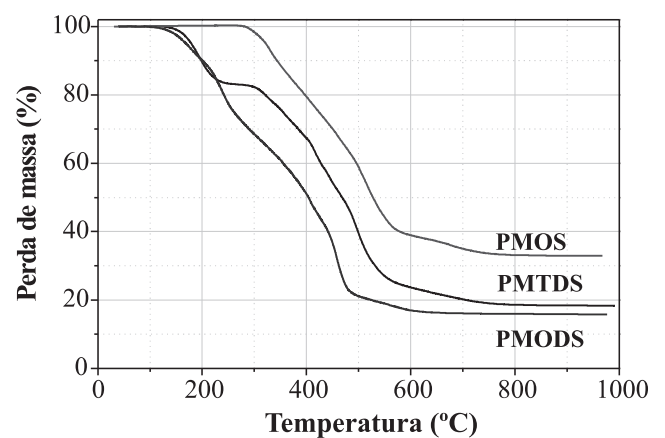

Figura 2. Curvas TG dos polímeros PMOS, PMTDS e PMODS em atmosfera oxidante

De acordo com a Figura 2, os três polissiloxanos são degradados quase totalmente na faixa de temperatura compreendida entre 150$650{ }^{\circ} \mathrm{C}$. O PMOS, polímero que possui a menor cadeia lateral, apresenta a maior estabilidade térmica entre os polissiloxanos estudados, o início de sua degradação ocorre somente a partir de $300{ }^{\circ} \mathrm{C}$. Após a degradação das cadeias orgânicas dos polímeros, uma pequena quantidade da massa inicial das amostras dos polímeros não foi volatilizada, aproximadamente 15\% de PMODS, $20 \%$ de PMTDS e $35 \%$ de PMOS. Este material resultante da combustão das amostras é constituído basicamente por sílica (Figura 1). Portanto, para a quantificação do conteúdo de carbono nas fases estacionárias devese levar em consideração apenas a faixa de temperatura de $150-650{ }^{\circ} \mathrm{C}$.

No entanto, perdas de massa provenientes dos suportes cromatográficos também podem influenciar o resultado final de porcentagem de carbono do material polimérico imobilizado nas fases estacionárias, devendo então ser eliminadas. Para quantificar esta contribuição, proveniente dos suportes cromatográficos, amostras em triplicadas de sílica nua e de sílica zirconizada foram analisadas por termogravimetria nas mesmas condições empregadas para análise dos polímeros. As curvas TG dos suportes estão apresentadas na Figura 3.

O perfil das curvas TG obtido para os dois suportes está de acordo com os normalmente obtidos na literatura para os mais diversos tipos de suportes de sílica ${ }^{7,11,12}$. Os dois materiais apresentaram perfis de degradação muito similares, nos quais as perdas de massa na re- 


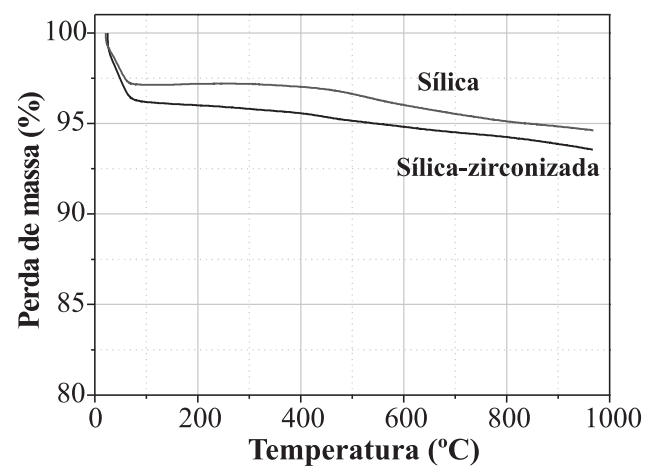

Figura 3. Curvas TG dos suportes de sílica Kromasil e sílica Kromasil zirconizada em atmosfera oxidante

gião de $25-150^{\circ} \mathrm{C}$ estão associadas à desidratação da superfície dos suportes cromatográficos, liberando moléculas de água fracamente adsorvidas. Entre 150 e $650{ }^{\circ} \mathrm{C}$ ocorre a condensação de grupos hidroxila superficiais (silanol e/ou zirconol), enquanto que acima de $650^{\circ} \mathrm{C}$ se inicia o processo de desidroxilação da superfície dos suportes, liberando moléculas de água. A perda total de massa dos suportes de sílica nua e sílica zirconizada é inferior a $7 \%$ do valor da massa inicial. Desta forma, na faixa de temperatura de $150-650{ }^{\circ} \mathrm{C}$, simultaneamente à degradação dos polissiloxanos, ocorre a condensação de grupos hidroxila dos suportes cromatográficos, perdendo massa referente ao desprendimento de moléculas de água. Esta quantidade de massa perdida deve ser subtraída da massa total perdida pelas fases estacionárias, nesta faixa de temperatura, no cálculo da porcentagem de carbono do material polimérico imobilizado. Depois de identificados os processos que resultaram em perdas de massa nos suportes cromatográficos e nos polímeros e determinadas as faixas de temperaturas características onde ocorre cada processo, foram realizadas as medidas por termogravimetria das quatro fases estacionárias avaliadas: $\mathrm{Si}(\mathrm{PMOS}), \mathrm{Si}(\mathrm{PMTDS})$ e $\mathrm{Si}(\mathrm{PMODS})$, nas quais o PMOS, o PMTDS e o PMODS, respectivamente, estão imobilizados termicamente sobre o suporte de sílica nua; e SiZr(PMTDS), na qual o PMTDS se encontra imobilizado termicamente sobre o suporte de sílica-zirconizada. As curvas TG obtidas para as fases estacionárias em estudo estão dispostas na Figura 4.

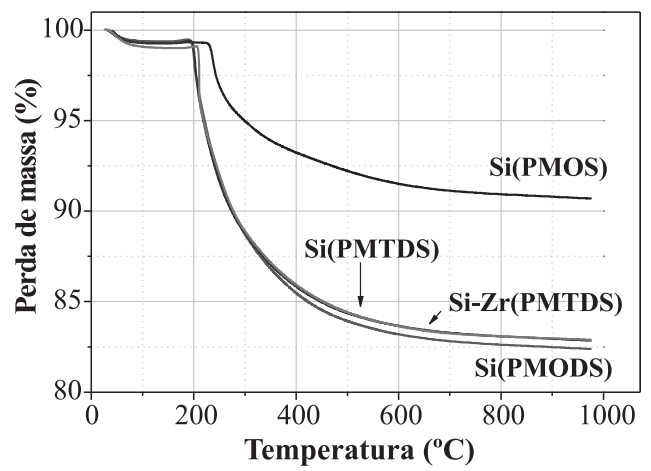

Figura 4. Curvas TG das fases estacionárias Si(PMOS), Si(PMTDS), Si(PMODS) e Si-Zr(PMTDS) em atmosfera oxidante

Os perfis das curvas TG obtidas para as fases estacionárias são bastante similares entre si. A fase $\mathrm{Si}(\mathrm{PMOS})$ apresentou uma perda de massa total menor que as demais fases estacionárias devido a menor quantidade de material orgânico imobilizado no suporte de sílica e, também, à estrutura química do PMOS que é constituída por monômeros com menor comprimento de cadeia carbônica. As fases $\mathrm{Si}(\mathrm{PMTDS})$ e $\mathrm{Si}-\mathrm{Zr}(\mathrm{PMTDS})$ apresentaram perfis de degradação praticamente idênticos, sugerindo que a influência do tipo do suporte cromatográfico nas medidas por TG é insignificante. A perda de massa da fase $\mathrm{Si}(\mathrm{PMODS})$, ligeiramente maior se comparada às demais fases estacionárias, está relacionada ao maior comprimento da cadeia carbônica do PMODS (com 18 átomos de carbono vs. 8 do PMOS e 14 do PMTDS), resultando em maior volatilização, na forma de $\mathrm{CO}_{2}$ e $\mathrm{H}_{2} \mathrm{O}$, do material polimérico adsorvido.

O perfil das curvas TG das fases estacionárias é compatível com os resultados de perdas de massa dos polímeros e dos suportes cromatográficos, na qual as maiores perdas estão associadas à degradação do polímero, entre $150-650^{\circ} \mathrm{C}$, restando uma quantidade acima de $80 \%$ do material após o término das medidas. Este fato se deve à presença dos suportes desidratados e desidroxilados $\left(\mathrm{SiO}_{2}\right)$, como afirmado anteriormente.

A porcentagem de carbono nas fases estacionárias pode ser determinada a partir dos seus valores de perdas de massa na faixa de temperatura de $150-650^{\circ} \mathrm{C}$, subtraindo deste valor as perdas provenientes dos suportes cromatográficos nesta faixa. A Equação 1 apresenta o cálculo da porcentagem de carbono baseado nos resultados fornecidos pela termogravimetria:

$\% C=\frac{\left(\% m_{T P}-\% m_{S P}\right) \% C_{p o l}}{\% m_{\text {inicial }}}$

onde: $\% m_{T P}=$ porcentagem de perda de massa da fase estacionária no intervalo de temperatura de 150 a $650{ }^{\circ} \mathrm{C} ; \% m_{S P}=$ porcentagem de massa perdida devido à condensação de grupos silanol e/ou zirconol da superfície do suporte entre 150 e $650{ }^{\circ} \mathrm{C} ; \% C_{p o l}=$ porcentagem de carbono presente em cada monômero; $\% m_{\text {inicial }}=$ porcentagem de massa de fase estacionária na temperatura de $150{ }^{\circ} \mathrm{C}$.

Empregando a Equação 1 foram determinadas as porcentagens de carbono para cada uma das fases estacionárias avaliadas, as quais se encontram dispostas na Tabela 2. Para efeito de comparação, a porcentagem de carbono das fases também foi determinada por análise elementar (Tabela 2). A relação entre os resultados de porcentagens de carbono obtidos para as fases estacionárias por termogravimetria e análise elementar foi investigada através da regressão linear (Figura 5).

Tabela 2. Porcentagens de carbono, obtidas por análise elementar e termogravimetria, e espessuras das camadas poliméricas das fases estacionárias em estudo

\begin{tabular}{lcccc}
\hline Amostras & $\% \mathrm{C}(\mathrm{CHN})^{\mathrm{a}}$ & $\% \mathrm{C}(\mathrm{TG})^{\mathrm{b}}$ & ${\text { Erro }(\%)^{\mathrm{c}}}$ & $\tau(\mathrm{nm})^{\mathrm{d}}$ \\
\hline Si(PMOS) & 6,14 & 6,40 & 4,23 & 0,47 \\
Si(PMTDS) & 14,18 & 14,11 & 0,50 & 1,00 \\
Si(PMODS) & 14,74 & 13,48 & 8,55 & 0,86 \\
Si-Zr(PMTDS) & 13,37 & 11,99 & 10,32 & 0,87 \\
\hline
\end{tabular}

${ }^{a}$ porcentagem de carbono das fases estacionárias obtidas por análise elementar; ${ }^{\mathrm{b}}$ porcentagem de carbono das fases estacionárias obtidas por termogravimetria; ${ }^{c}$ erro relativo calculado a partir da equação: | $\frac{[\% C(C H N)-\% C(T G)]}{\% C(C H N)} 100 \%$ |; d espessura das camadas poliméricas baseadas nas porcentagens de carbono obtidas por TG.

Pode ser observado que as porcentagens de carbono obtidas para as fases estacionárias pelas técnicas são bastante similares, mostrando uma boa correlação entre seus resultados, coeficiente de correlação (r) igual a 0,985. Além disso, as médias de porcentagens de carbono obtidas para cada fase estacionária pelos dois métodos 


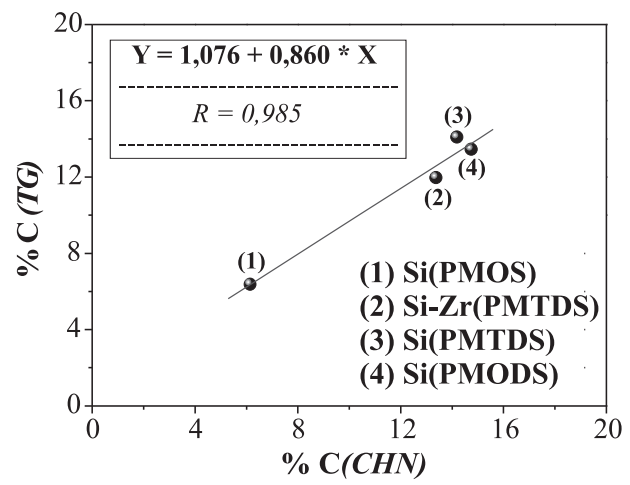

Figura 5. Relação entre as porcentagens de carbono obtidas pela análise elementar (método convencional) e por termogravimetria

foram estatisticamente equivalentes no nível de $95 \%$ de probabilidade pelo teste $t$-Student. Logo, o método termogravimétrico possui eficiência adequada para a determinação da porcentagem de carbono de fases estacionárias baseadas na imobilização térmica de polímeros sobre suportes cromatográficos.

A quantidade de material orgânico ligado ou imobilizado nas fases estacionárias normalmente é expressa em termos da concentração superficial do ligante ou da espessura da camada polimérica imobilizada $(\tau)$, dependendo do tipo de fase estacionária avaliada. Para a conversão dos valores de porcentagem de carbono em concentração do ligante nas fases estacionárias contendo polímeros imobilizados sobre suportes é utilizada a Equação 2. Para tal, é necessário o conhecimento da área superficial específica dos suportes cromatográficos em estudo e, também, considerar que há a formação de uma fina camada polimérica em toda a extensão da superfície do suporte, inclusive nas paredes internas de seus poros, eventualmente podendo preenchê-los completamente caso o diâmetro do poro seja inferior a 2 vezes a espessura da camada polimérica.

$$
X=\frac{\% C \times M_{p o l}}{12 \times 100 n_{C} \times S_{B E T}\left[1-\frac{\% C\left(M_{p o l}-1\right)}{12 \times 100 n_{C}}\right]}
$$

onde: $X=$ concentração, em $\mathrm{g} \mathrm{m}^{-2}$, do polímero imobilizado sobre o suporte; $\% C=$ percentagem de carbono da fase estacionária calculada pela Equação 1; $M_{p o l}=$ massa molar de cada monômero do polímero; $n_{C}=$ número de átomos de carbono presentes na cadeia lateral do monômero; $S_{B E T}=$ área superficial específica do suporte cromatográfico; 12 = massa molar do átomo de carbono.

Esta concentração do ligante na superfície e poros do suporte pode ser facilmente convertida em espessura de camada polimérica, conhecendo-se o valor de densidade do polímero em estudo - informação normalmente fornecida pelo fabricante. A relação entre a concentração e a espessura de camada do polímero está apresentada na Equação 3.

$$
\tau=\frac{X}{\rho} 10^{-6}
$$

onde: $\tau=$ espessura da camada polimérica imobilizada na superfície e nos poros do suporte cromatográfico $(\mathrm{em} \mathrm{nm}) ; \rho=$ densidade específica do polímero; $10^{-6}=$ correção de unidades.

As espessuras das camadas de PMOS, PMTDS e PMODS nas fases estacionárias estudadas de Si(PMOS), Si(PMTDS), $\mathrm{Si}(\mathrm{PMODS})$ e $\mathrm{Si}-\mathrm{Zr}(\mathrm{PMTDS})$ foram calculadas a partir das equações 2 e 3. Os resultados obtidos estão apresentados na Tabela 2.

\section{CONCLUSÕES}

A metodologia descrita neste trabalho comprova que a termogravimetria pode ser empregada com eficiência na determinação da espessura da camada polimérica de fases estacionárias preparadas em laboratório, principalmente para fases baseadas na imobilização de polissiloxanos sobre suportes de sílica e sílica metalizada. Quatro fases estacionárias contendo polímeros de diferentes comprimentos de cadeia lateral foram analisadas e os resultados obtidos em termos de porcentagem de carbono foram bastante similares aos obtidos pelo método convencional, análise elementar. $\mathrm{O}$ procedimento analítico desenvolvido mostrou-se bastante simples e efetivo, resultando em porcentagens de carbono com bastante precisão para as fases estacionárias estudadas, podendo ser aplicado a fases contendo uma vasta gama de diferentes polímeros imobilizados sobre suportes cromatográficos.

\section{AGRADECIMENTOS}

À Fundação de Amparo à Pesquisa do Estado de São Paulo (FAPESP) e ao Conselho Nacional de Pesquisa e Desenvolvimento Tecnológico (CNPq) pelas bolsas e suporte financeiro.

\section{REFERÊNCIAS}

1. Kirkland, J. J.; J. Chromatogr, A 2004, 1060, 9.

2. Jardim, I. C. S. F.; Collins, K. E.; Collins, C. H.; Microchem. J. 2004, 77, 191.

3. Faria, A. M.; Jardim, I. C. S. F.; Collins, K. E.; Collins, C. H.; J. Sep. Sci. 2006, 29, 782.

4. Pesek, J. J.; Matyska, M. T.; Interface Sci. 1997, 5, 103.

5. Kobayashi, S.; Tanaka, I.; Shirota, O.; Kanda, T.; Ohtsu, Y.; J. Chromatogr, A 1998, 828, 75.

6. Sakaki, K.; Sudo, Y.; Kudo, S.; J. Liq. Chromatogr. Relat. Technol. 2004 , $27,387$.

7. Zhuravlev, L. T.; Colloids Surf., A 2000, 173, 1.

8. Schilling, M. R.; Preusser, F.; Gutnikov, G.; J. Thermal Anal. 1992, 38, 2483.

9. Lumley, B.; Khong, T. M.; Perrett, D.; Chromatographia 2004, 60, 59.

10. Faria, A. M.; Magalhães, D. R.; Collins, K. E.; Collins, C. H.; Anal. Chim. Acta 2005, 550, 137.

11. Shalliker, R. A.; Rintoul, L.; Douglas, G. K.; Russell, S. C.; J. Mater. Sci. 1997, 32, 2949.

12. Alekseev, S. A.; Zaitsev, V. N.; Chem. Mater. 2006, 18, 1981. 